Published in final edited form as:

Clin Cancer Res. 2008 March 1; 14(5): 1349-1354. doi:10.1158/1078-0432.CCR-07-4110.

\title{
Evaluation of Colon Cancer - Specific Antigen 2 as a Potential Serum Marker for Colorectal Cancer
}

\author{
Eddy S. Leman ${ }^{1}$, Robert E. Schoen ${ }^{3,4}$, Ahmed Magheli ${ }^{1}$, Lori J. Sokoll ${ }^{1,2}$, Daniel W. Chan ${ }^{1,2}$, \\ and Robert H. Getzenberg ${ }^{1,2}$
}

${ }^{1}$ The Brady Urological Institute, Johns Hopkins University School of Medicine, Baltimore, Maryland ${ }^{2}$ Department of Pathology, Johns Hopkins University School of Medicine, Baltimore, Maryland ${ }^{3}$ Division of Gastroenterology, Hepatology, and Nutrition, University of Pittsburgh and the University of Pittsburgh Cancer Institute, Pittsburgh, Pennsylvania ${ }^{4}$ Department of Epidemiology, University of Pittsburgh and the University of Pittsburgh Cancer Institute, Pittsburgh, Pennsylvania

\section{Abstract \\ Purpose-A blood test to detect colon cancer at a preventable stage would represent a major advancement. We have previously identified colon cancer - specific markers using focused proteomics analysis of nuclear structural proteins. Two of these markers, colon cancer - specific antigen (CCSA)-3 and CCSA-4, have been developed into blood-based markers that are able to distinguish individuals with colorectal cancer from those without. CCSA-2 is a distinct novel colon cancer marker identified using focused proteomics.}

Experimental Design-Using an indirect ELISA on serum samples obtained from two institutions, we evaluated CCSA-2 as a serum-based colon cancer marker. A total of111serumsamples from individuals who underwent colonoscopy and were subsequently diagnosed as either being normal or having hyperplastic polyps, nonadvanced adenomas, advanced adenomas, and colorectal cancer were evaluated. A diverse control population that consisted of 125 serum samples was also included in this study.

Results-Receiver operating characteristic analyses were used to measure the sensitivity and specificity of CCSA-2. CCSA-2 at a cutoff of $10.8 \mu \mathrm{g} / \mathrm{mL}$ has overall specificity of $78.4 \%$ [95\% confidence interval $(95 \% \mathrm{CI}), 67.3-87.1 \%$ ] and sensitivity of $97.3 \%$ (95\% CI, 85.8-99.5\%) in separating individuals with advanced adenomas and colorectal cancer from normal, hyperplastic, and nonadvanced adenoma populations. The receiver operating characteristic curve for CCSA-2 has an area under the curve of 0.90 (95\% CI, 0.83-0.95).

Conclusion-Our initial study shows that CCSA-2 is a potential serum-based marker for colon cancer detection with high sensitivity and specificity.

Requests for reprints: Robert H. Getzenberg, The Brady Urological Institute, Johns Hopkins Hospital, Marburg 121, 600 North Wolfe Street, Baltimore, MD 21287. Phone: 410-502-3137; Fax: 410-502-9336; rgetzen1@jhmi.edu.

Note: Supplementary data for this article are available at Clinical Cancer Research Online (http://clincancerres.aacrjournals.org/). 
Colorectal cancer is one of the most common cancers worldwide, and it is the third most diagnosed cancer in the United States. In 2007, American Cancer Society estimates that there will be 153,760 new cases and 52,180 related deaths for colon cancer (1). The basis for colon cancer development typically is considered to involve the evolution of adenomatous polyps to carcinoma $(2,3)$. Individuals with a history of adenomatous polyps have a higher risk of subsequent cancer (4). Removal of adenomatous polyps results in a reduction in colon cancer incidence (5). The foundation for pursuing screening for colon cancer is that detection of early-stage cancer as well as the adenomatous polyps may help reduce the risk of the disease.

Several methods are available for the detection of colon cancer. These include fecal occult blood testing, flexible sigmoidoscopy, double contrast barium enema, as well as colonoscopy (6). However, the costs and risk of complications and discomfort have made these tests not as widely used by general public. For example, although the fecal occult blood test is noninvasive, it is not sensitive for detection of adenomatous polyps. Fecal occult blood test may produce false-positive results because blood may be present in the stool for other reasons, such as hemorrhoidal bleeding. Although colonoscopy results in high sensitivity (7), this screening method is invasive. A noninvasive biological marker would be of great benefit.

One of the signatures of a cancer cell is changes in the nuclear structure and architecture. In concordance with these changes are alterations in the composition of nuclear structural proteins. Alterations in these proteins are associated with various types of cancer such as breast, prostate, bladder, lung, ovarian, as well as squamous carcinoma of the neck $(8,9)$. Our laboratory has used the proteomics approach to identify a set of proteins associated with changes in the nuclear structure/architecture. Assessment of these nuclear proteins resulted in identification of biomarkers that are specific for colon cancer: colon cancer-specific antigen (CCSA)-2, CCSA-3, and CCSA-4 (10). These proteins are associated with colon cancer tissue and are not found in normal adjacent tissue or the normal colon (10). Recently, we showed that both CCSA- 3 and CCSA- 4 can be used as highly specific and sensitive serum-based markers for detecting individuals with colon cancer and separating them from those with other benign diseases and cancer types as well as normal individuals (11).

Using a similar approach, we now show that CCSA-2 can also serve as another potential serum-based marker for detection of colorectal cancer. An antibody against CCSA-2 was used to develop a serum-based ELISA and then used to evaluate samples from individuals presenting for colonoscopy, with a variety of outcomes, along with samples from individuals with other cancer types and benign conditions. The main objective of this study is to examine the performance characteristics of CCSA-2 immunoassays for detection of colorectal cancer in these selected populations. Similar to our previous markers, these studies examine the sensitivity and specificity values of CCSA-2 in relevant clinical populations and are not necessarily reflective of a screening population. 


\section{Materials and Methods}

\section{Sequencing and antibody production of CCSA-2}

The CCSA-2 protein (MW 56,000 Da, isoelectric point 6.22) was isolated from twodimensional gels and the spot was then concentrated and subjected to $\mathrm{NH}_{2}$-terminal protein sequencing. Using the peptide sequence derived from the CCSA-2 epitopes, peptides and polyclonal antibodies for these epitopes were produced. Antibodies for CCSA-2 were made by Cocalico Biologicals, Inc., under an Institutional Animal Care and Use Committeeapproved protocol.

\section{Populations and samples}

The first group of serum samples $(n=101)$ were collected from consented patients under an Institutional Review Board-approved protocol from the University of Pittsburgh Medical Center (UPMC; Pittsburgh, PA). These individuals underwent colonoscopy or colon cancer surgery, and they participated as part of the National Cancer Institute Early Detection Research Network. For subjects undergoing colonoscopy, samples were generally drawn from a fasting participant, immediately before the colonoscopy procedure, and thus before knowledge of the colonoscopy result. An adenoma was defined as advanced if it contained villous features (villous or tubulovillous adenomas), was large ( $\geq 1 \mathrm{~cm}$ as measured by the pathologist, or by the endoscopist when removed in a piecemeal fashion), or had high-grade dysplasia. Based on the histologic results of the colonoscopy, subjects were classified into one of four mutually exclusive, hierarchical categories: normal colonoscopy $(n=25)$, hyperplastic polyp $(n=18)$, nonadvanced adenoma $(n=31)$, and advanced adenoma $(n=$ 14). To increase recruitment of subjects with advanced adenoma, some subjects with polyps $\geq 1.0 \mathrm{~cm}$ were recruited and blood was drawn after the colonoscopy procedure was completed and the effect of the conscious sedation had abated. More than $92 \%$ of subjects with normal, hyperplastic, or nonadvanced adenoma were drawn pre-procedure. Eight of 18 subjects with an advanced adenoma were drawn post-procedure, usually within 45 minutes of the completion of the colonoscopy. Of the 13 cancer subjects recruited at UPMC, 7 had colon cancer and 6 had rectal cancer; 1 had blood drawn pre-colonoscopy and 12 were drawn preoperatively in the holding area immediately before the cancer surgery. Samples were stored at $-80^{\circ} \mathrm{C}$ before analysis.

The second set of sera $(n=135)$ were collected from the Johns Hopkins Hospital (JHH). This set of sera consisted of 10 individuals who were diagnosed with nonmetastatic colon cancer, as well as 125 samples from individuals with other benign diseases and cancer types. In subjects who underwent surgery, these samples were collected before surgery and processed promptly after collection and stored at $4{ }^{\circ} \mathrm{C}$ for a maximum of 48 hours before freezing at $-70^{\circ} \mathrm{C}$. Both genders are well represented and, as expected, subjects are in an appropriate age range for colon testing (Table 1). There was a relatively high rate of family history of colorectal cancer in first-degree relatives in subjects with the normal and hyperplastic polyps on colonoscopy (Table 1$)$. Stages of colorectal cancer were well represented: stage I $(n=4)$, stage II $(n=8)$, stage III $(n=8)$, and stage IV $(n=3)$. 


\section{CCSA-2 indirect ELISA}

A set of serum samples $(n=24)$ from consented patients under an Institutional Review

Board-approved protocol from UPMC were used in the pilot studies. These pilot sera from normal individuals $(n=5)$, individuals with hyperplastic polyps $(n=5)$, nonadvanced adenomas $(n=5)$, advanced adenomas (4), as well as those with colorectal cancer $(n=5)$ were used to evaluate the indirect ELISA assay for CCSA-2, using an anti-epitope antibody against CCSA-2.2. Once the conditions and cutoff points were reproducible from assay to assay, the study was expanded to additional individuals. The coefficient of variation on repeat testing of the same samples on three separate runs in duplicate was within $10 \%$. The serum samples used in the pilot studies were not included in our subsequent analyses of CCSA-2 in the serum.

Serum samples were plated in duplicates and in random order using 96-well Nunc Immunoplate MaxiSorb plates for overnight at room temperature. On each plate, samples for each of the patient groups were run and these were distributed across the plates. Samples were aspirated and blocked in Super Block (Pierce). The blocking buffer was removed and then washed with $1 \times$ TBS with $0.05 \%$ Tween 20 (TBS-T). Polyclonal antibody for CCSA-2 was added and incubated for 2 hours at $37^{\circ} \mathrm{C}$. The plates were rinsed with TBS-T and secondary goat antirabbit peroxidase antibody (KPL) was added and incubated at $37^{\circ} \mathrm{C}$ for 2 hours. The plates were washed with TBS-T, followed by incubation with room temperature TMB substrate (KPL) for 30 seconds. The plates were read at $630 \mathrm{~nm}$ using Pherastar plate reader (BMG Lab Tech). Inter-plate and intra-assay variability for each assay was verified by plating various concentrations of the CCSA-2 peptide $(0-1 \mathrm{mg} / \mathrm{mL})$ and rabbit IgG in duplicates in each plate. To estimate the concentration of each sample, various concentrations $(0.0,0.1,1,10,100,1,000$, and $10,000 \mathrm{ng} / \mathrm{mL})$ of peptide control for CCSA-2 were fitted on a sigmoidal dose-response curve using GraphPad Prism 4 software, and the concentration was calculated from the equation generated by the curve.

\section{Statistical analysis}

We constructed receiver operating characteristic (ROC) curves and determined the area under the curve to show the ability of CCSA-2 to differentiate nonneoplastic (normal, hyperplastic, and nonadvanced advanced adenoma) from neoplastic or potentially neoplastic lesions (colorectal cancer or advanced adenoma). All statistical analyses (two-tailed; $P<$ 0.05 was considered significant) as well as upper and lower bounds for confidence intervals for sensitivity and specificity were calculated by exact methods based on the binomial distribution using MedCalc (version 9.210) and SPSS (version 15.0, SPSS).

\section{Results}

The specificity of CCSA-2 antibody used in these studies was first examined by testing its cross-reactivity with several proteins such as early prostate cancer antigen and early prostate cancer antigen-2 (both of which are prostate cancer-specific nuclear matrix proteins), as well as CCSA-3 and CCSA-4, both of which are colon cancer-associated nuclear structural proteins that are different and not related to CCSA-2. As shown in Fig. 1, CCSA-2 did not 
react with any of these proteins, indicating that antibody against CCSA-2 was specific for this antigen.

Pilot studies using 24 sera samples from normal-normal individuals, normal-hyperplastic individuals, individuals with nonadvanced and advanced adenomas, as well as those with colon cancer were evaluated with the CCSA-2 assay. The pilot assays for CCSA-2 clearly showed that colon cancer population was separated from those with adenomas and those without the disease (Supplementary Fig. S1). The cutoff point for CCSA-2 at $10.8 \mu \mathrm{g} / \mathrm{mL}$ was determined based on the pilot data. At the time these studies were done, the recombinant protein for CCSA-2 was not available. Thus, the concentrations that correspond to the absorbance levels were estimated by the equation that resulted from fitting various peptide concentrations to a sigmoidal curve. On completion of the pilot studies, inter-assay variability was measured by running repeated samples on the assays. The studies showed values that are within $10 \%$ of one another. Using the established cutoff point, the CCSA-2 assays were tested on the larger sample populations $(n=236)$ that did not include samples from the pilot study.

The mean levels of CCSA-2 for each sample population are presented in Table 1, and the individual values on a linear scale are represented in dot-plot graphs (Fig. 2). The sensitivity and specificity of CCSA-2 assay are summarized in Table 2. CCSA-2 assay at $10.8 \mu \mathrm{g} / \mathrm{mL}$ has a sensitivity of $95.6 \%$ [95\% confidence interval (95\% CI), 78.1-99.8\%] in detecting individuals with colorectal cancer. When individuals with advanced adenoma and colorectal cancer are grouped together, the sensitivity of CCSA-2 assay is $97.3 \%$ (95\% CI, 85.899.5\%). At the same cutoff, CCSA-2 assay has a specificity of $97.7 \%$ (95\% CI, 873.799.9\%) in separating normal and hyperplastic populations from all others (Table 2). When individuals with nonadvanced adenomas are taken into account, the CCSA-2 assay has a specificity of 78.4\% (95\% CI, 67.3-87.1\%). In addition, CCSA-2 assay has a specificity of $100 \%$ in separating the benign disease populations and those with other types of cancer from those with advanced adenoma and colon cancer.

The sensitivity and specificity values of the CCSA-2 assay were further analyzed by performing ROC analyses. As shown in Fig. 3, the area under the curve for CCSA-2 is 0.90 (95\% CI, 0.83-0.95; $P<0.0001$ ) in separating individuals with normal, hyperplastic, and nonadvanced adenoma conditions from those with advanced adenomas and colorectal cancers. The ROC curve also provides several cutoff points (A-F) to show the trade-off between sensitivity and specificity at different cutoff values. As shown in the curve, cutoff point $E$ corresponds with our selected cutoff at $10.8 \mathrm{mg} / \mathrm{mL}$, resulting in $97.3 \%$ sensitivity (95\% CI, 85.8-99.5\%) and 78.4\% specificity (95\% CI, 67.3-87.1\%). When the cutoff point is increased to $11.05 \mu \mathrm{g} / \mathrm{mL}$ (point $\mathrm{D}$ ), the sensitivity decreases to $86.5 \%$, whereas the specificity increases slightly to $82.4 \%$. Lowering the cutoff point to $10.27 \mu \mathrm{g} / \mathrm{mL}$ (point F) results in $100 \%$ sensitivity, but lower specificity (48.7\%).

There was a significant association between adenoma/polyp size and CCSA-2 levels. As shown in Fig. 4, ROC analyses of CCSA-2 in individuals with larger polyps (advanced adenomas) versus those with smaller polyps (nonadvanced adenomas) show an area under the curve of 0.83 (95\% CI, 0.69-0.92). In addition, Spearman's analyses (Supplementary 
Fig. S2) also show that individuals with larger polyps have higher levels of CCSA-2 (Spearman's coefficient $=0.575 ; P<0.0001$ ). . However, there was no significant association between cancer stage and CCSA-2 concentration (results not shown). In control populations without evidence of colorectal neoplasia (UPMC normal and hyperplastic polyp, JHH benign disease, and JHH other cancer; total $n=168$ ), CCSA-2 distribution was not statistically different in individuals younger than 60 years versus 60 years or older. The male population showed higher CCSA-2 values than female (median, 9.82 versus $9.37 \mu \mathrm{g} / \mathrm{mL} ; P$ $=0.034$ ). In cancer-free subjects from UPMC (normal and hyperplastic; total $n=43$ ), CCSA-2 distribution did not differ according to history of polyps or personal history of cancer. There was also no significant difference in median CCSA-2 values in individuals with than in those without a family cancer history (CCSA-2, 9.39 versus $9.73 \mu \mathrm{g} / \mathrm{mL} ; P=$ 0.693).

\section{Discussion}

We have previously identified six colon cancer markers with a focused-proteomic approach (CCSA-1 to CCSA-6; ref. 10). Two of these markers, CCSA-3 and CCSA-4, have recently been developed as serum-based markers for the detection of colon cancer. The purpose of our present study is to examine whether another marker, CCSA-2, can also be used as a highly specific and sensitive serum-based marker for colon cancer detection. The mechanisms by which CCSA-2 is found in the serum are currently under investigation. We speculate that this protein is released into the blood by cellular breakdown or apoptosis and is quite stable once it gets there (12).

The CCSA-2 immunoassays show an increase in mean values from normal and hyperplastic populations to nonadvanced adenomatous pathology with a further increase in advanced adenoma and cancer. These values reflect the pathologic development of disease along the adenoma-carcinoma pathway. Our recently characterized immunoassays for colon cancer serum markers CCSA-3 and CCSA-4 had a combined sensitivity of $97.3 \%$ and a specificity of $78.1 \%$ in separating individuals with colon cancer and advanced adenomas from those who were classified as having normal, hyperplastic, and nonadvanced adenomas (11). However, there was no correlation between adenoma size and the levels of either CCSA-3 or CCSA-4 in the serum (11). The CCSA-2 immunoassays have higher sensitivity (97.3\%) and slightly lower specificity (78.4\%) than the CCSA-3 and CCSA-4 combined immunoassays. The CCSA-2 immunoassays are unique from CCSA-3 and CCSA-4 in that they show that individuals with advanced adenomas have higher levels of CCSA-2 in their blood than those with nonadvanced adenomas [area under the curve of 0.83 (95\% CI, 0.69-0.92) and Spearman's correlation coefficient of $0.479 ; P<0.001]$. Our results correlate with our previous tissue analyses in which CCSA-2 was found only in malignancy, but not in any of the premalignant polyps (13). In addition, CCSA-2 expression was found in the junction of advanced adenomas and $80 \%$ of invasive cancer, suggesting that CCSA- 2 was expressed at point of invasion (13).

Taken together, the findings presented in this study show that CCSA-2, another colon cancer-associated nuclear protein, can be used as a potential serum-based marker for colon cancer detection. The CCSA-2 immunoassay results represent characterization of an assay, 
and it is not designed to determine the performance of this assay in screening patients for colorectal cancer. In addition, the populations studied in this article are not representative of distributions found in a screening population. Future studies should, as best possible, evaluate an unselected screening population. Whereas further validation studies are clearly warranted, to our knowledge, the data provided in this report may serve as groundwork for the development of a blood-based assay for colon cancer screening.

\section{Supplementary Material}

Refer to Web version on PubMed Central for supplementary material.

\section{Acknowledgments}

Grant support: NIH, National Cancer Institute grant CA084968 and Onconome, Inc., and the Patana Fund of the Brady Urological Institute.

\section{References}

1. Jemal A, Siege IR, Ward E, Murray T, Xu J, Thun MJ. Cancer statistics, 2007. CA Cancer J Clin. 2007; 57:43-66. [PubMed: 17237035]

2. Bond JH. Clinical evidence for the adenoma-carcinoma sequence, and the management of patients with colorectal adenomas. Semin Gastrointest Dis. 2000; 11:176-184. [PubMed: 11057945]

3. Leslie A, Carey FA, Pratt NR, Steele RJ. The colorectal adenoma-carcinoma sequence. Br J Surg. 2002; 89:845-860. [PubMed: 12081733]

4. Atkin WS, Morson BC, Cuzick J. Long-term risk of colorectal cancer after excision of rectosigmoid adenomas. N Engl J Med. 1992; 326:658-662. [PubMed: 1736104]

5. Mandel JS, Church TR, Ederer F, Bond JH. Colorectal cancer mortality: effectiveness of biennial screening for fecal occult blood. J Natl Cancer Inst. 1999; 91:434-437. [PubMed: 10070942]

6. Hawk ET, Levin B. Colorectal cancer prevention. J Clin Oncol. 2005; 23:378-391. [PubMed: 15637400]

7. Smith RA, Cokkinides V, von Eschenbach AC, et al. American Cancer Society guidelines for the early detection of cancer. CA Cancer J Clin. 2002; 52:8-22. [PubMed: 11814067]

8. Konety BR, Getzenberg RH. Nuclear structural proteins as biomarkers of cancer. J Cell Biochem. 1999; (Suppl 32-33):183-191. [PubMed: 10629118]

9. Leman ES, Getzenberg RH. Nuclear structure as a source of cancer specific biomarkers. J Cell Biochem. Epub 2007 Apr 23.

10. Brunagel G, Vietmeier BN, Bauer AJ, Schoen RE, Getzenberg RH. Identification of nuclear matrix protein alterations associated with human colon cancer. Cancer Res. 2002; 62:2437-2442. [PubMed: 11956108]

11. Leman ES, Schoen RE, Weissfeld JL, et al. Initial analyses of colon cancer-specific antigen (CCSA)-3 and CCSA-4 as colorectal cancer-associated serum markers. Cancer Res. 2007; 67:5600-5605. [PubMed: 17575123]

12. Miller TE, Beausang LA, Winchell LF, Lidgard GP. Detection of nuclear matrix proteins in serum from cancer patients. Cancer Res. 1992; 52:422-427. [PubMed: 1728414]

13. Brunagel G, Schoen RE, Getzenberg RH. Colon cancer specific nuclear matrix protein alterations in human colonic adenomatous polyps. J Cell Biochem. 2004; 91:365-374. [PubMed: 14743395] 


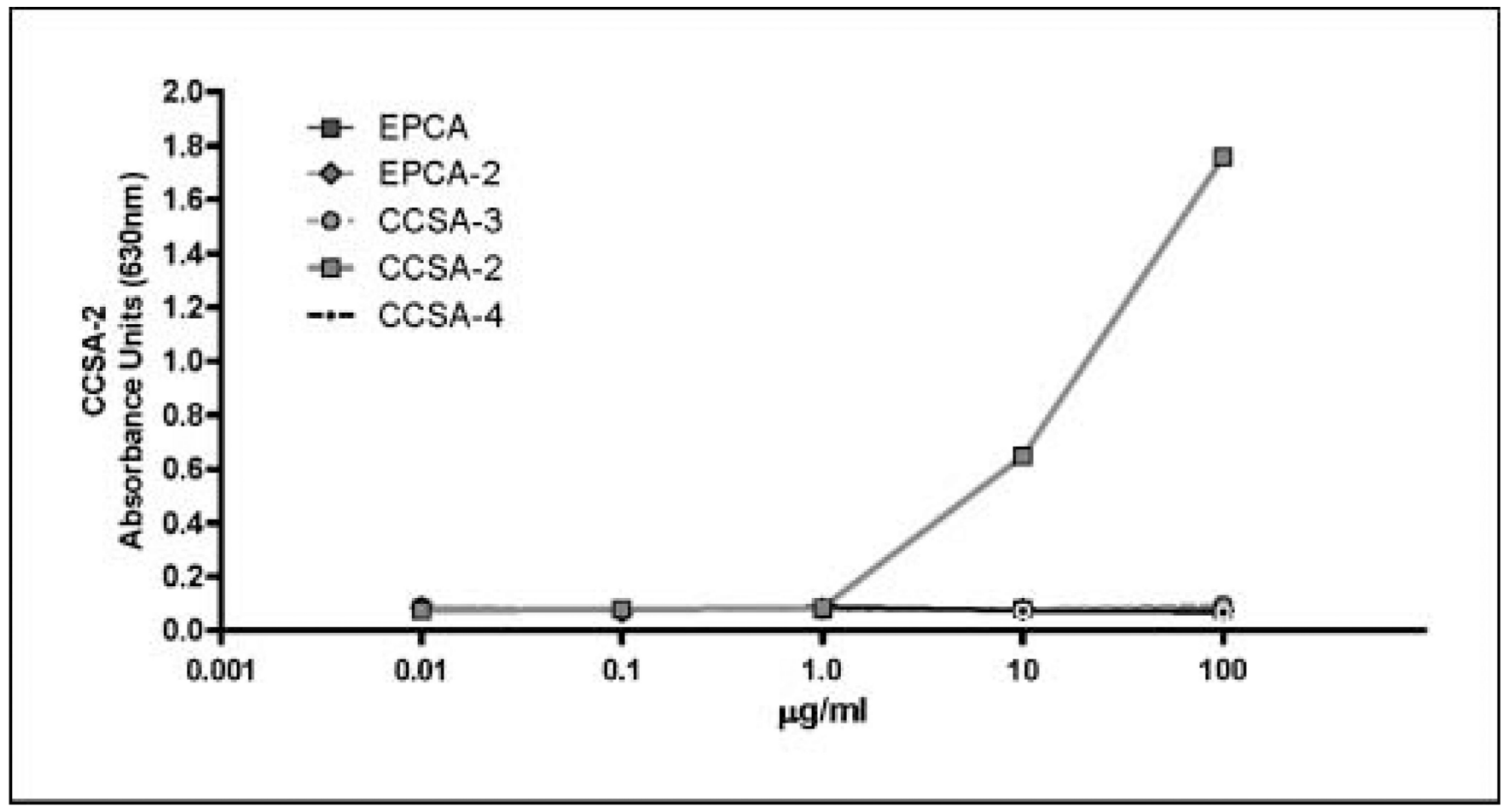

Fig. 1.

Specificity of CCSA-2 antibodies. The CCSA-2 antibody only reacts with the CCSA-2 peptide but not with the other peptides [early prostate cancer antigen (EPCA), early prostate cancer antigen-2 (EPCA-2), CCSA-3, and CCSA-4]. 


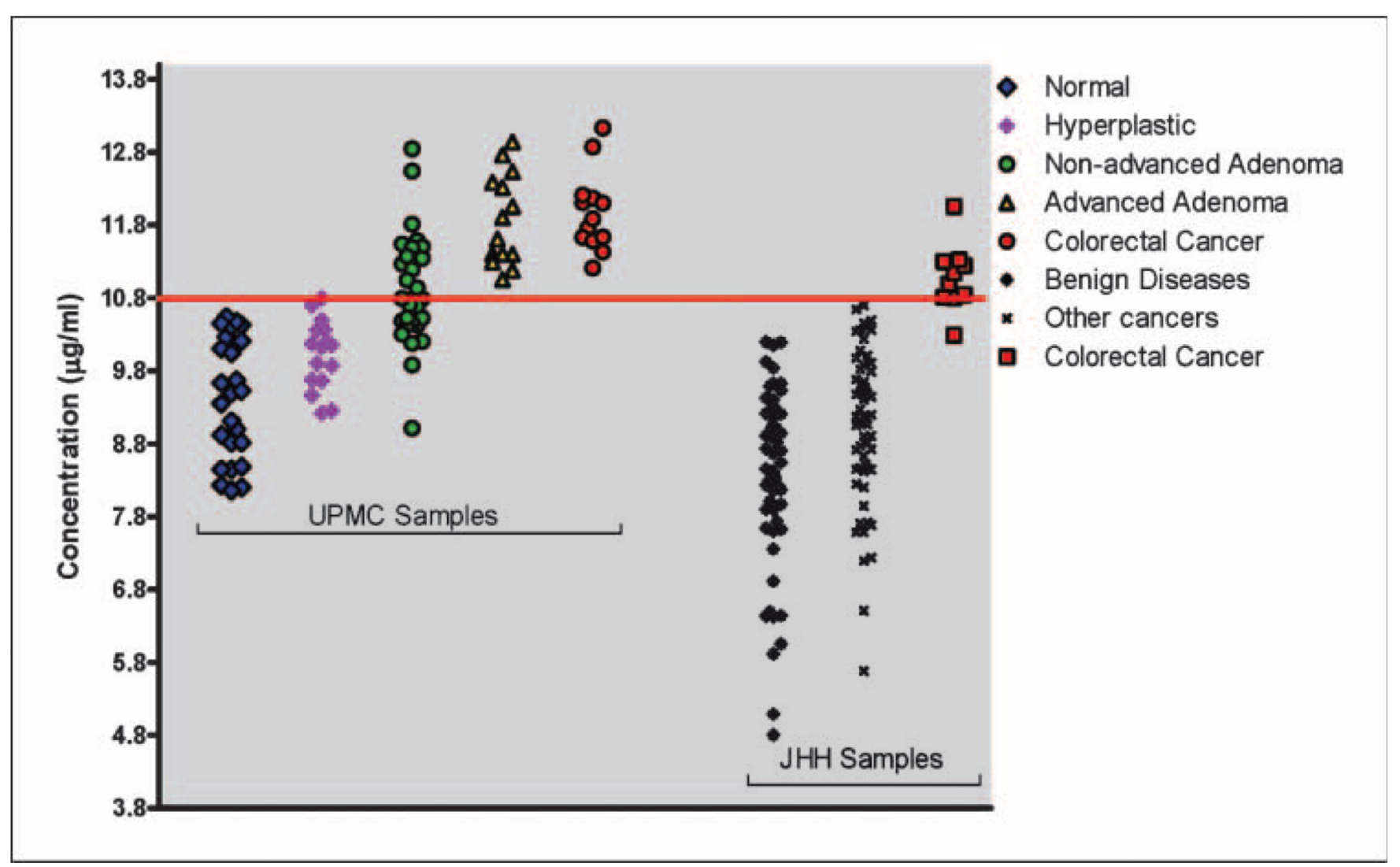

Fig. 2.

Using serum samples collected from 236 individuals, indirect ELISA assays show that CCSA-2 has a cutoff point of $\geq 10.8 \mu \mathrm{g} / \mathrm{mL}$ in separating normal, hyperplastic, and benign individuals from cancer populations. 


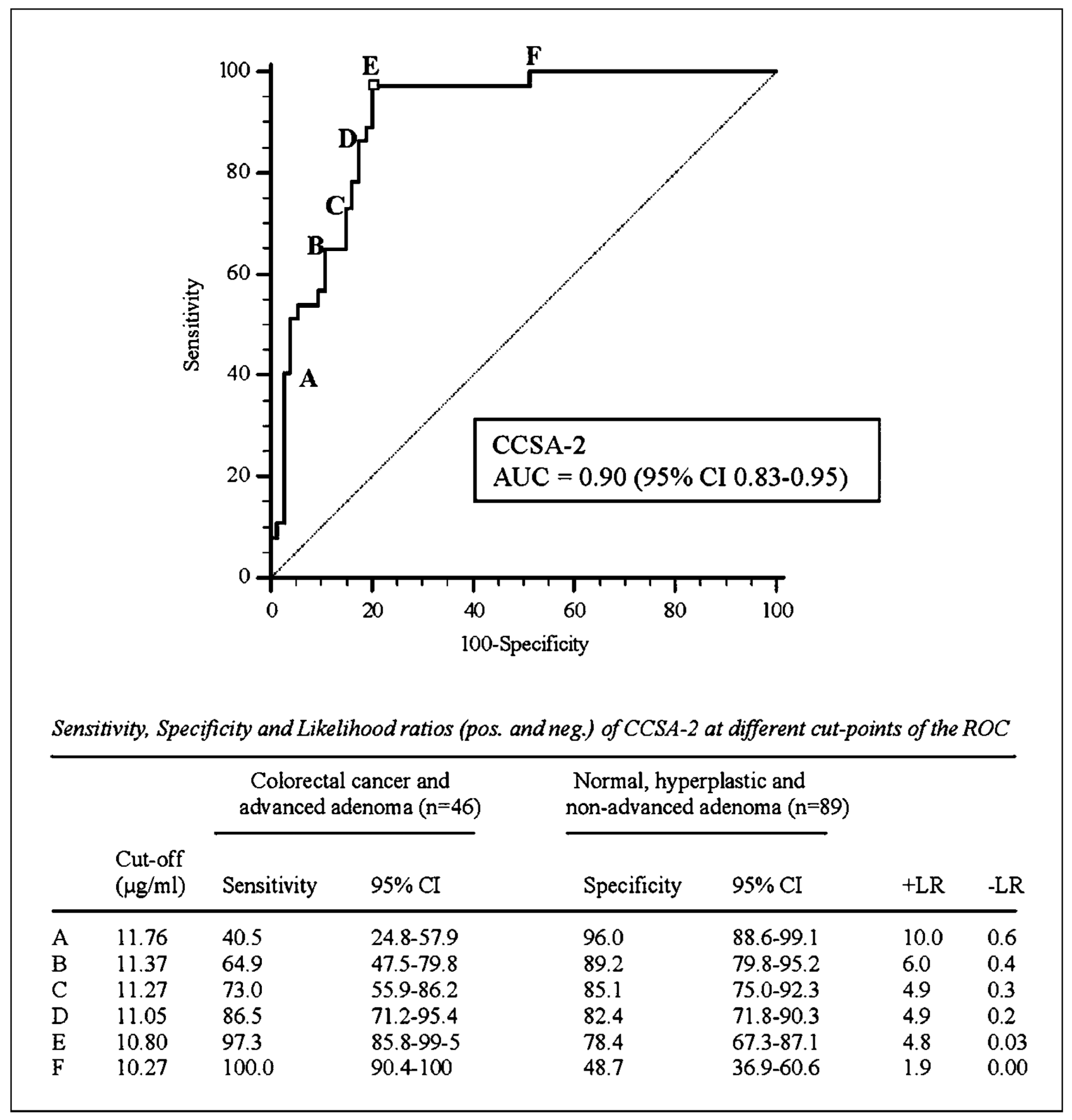

Fig. 3.

ROC curves forCCSA-2inseparating normal, hyperplastic, and benign individuals from advanced adenoma and cancer populations. 


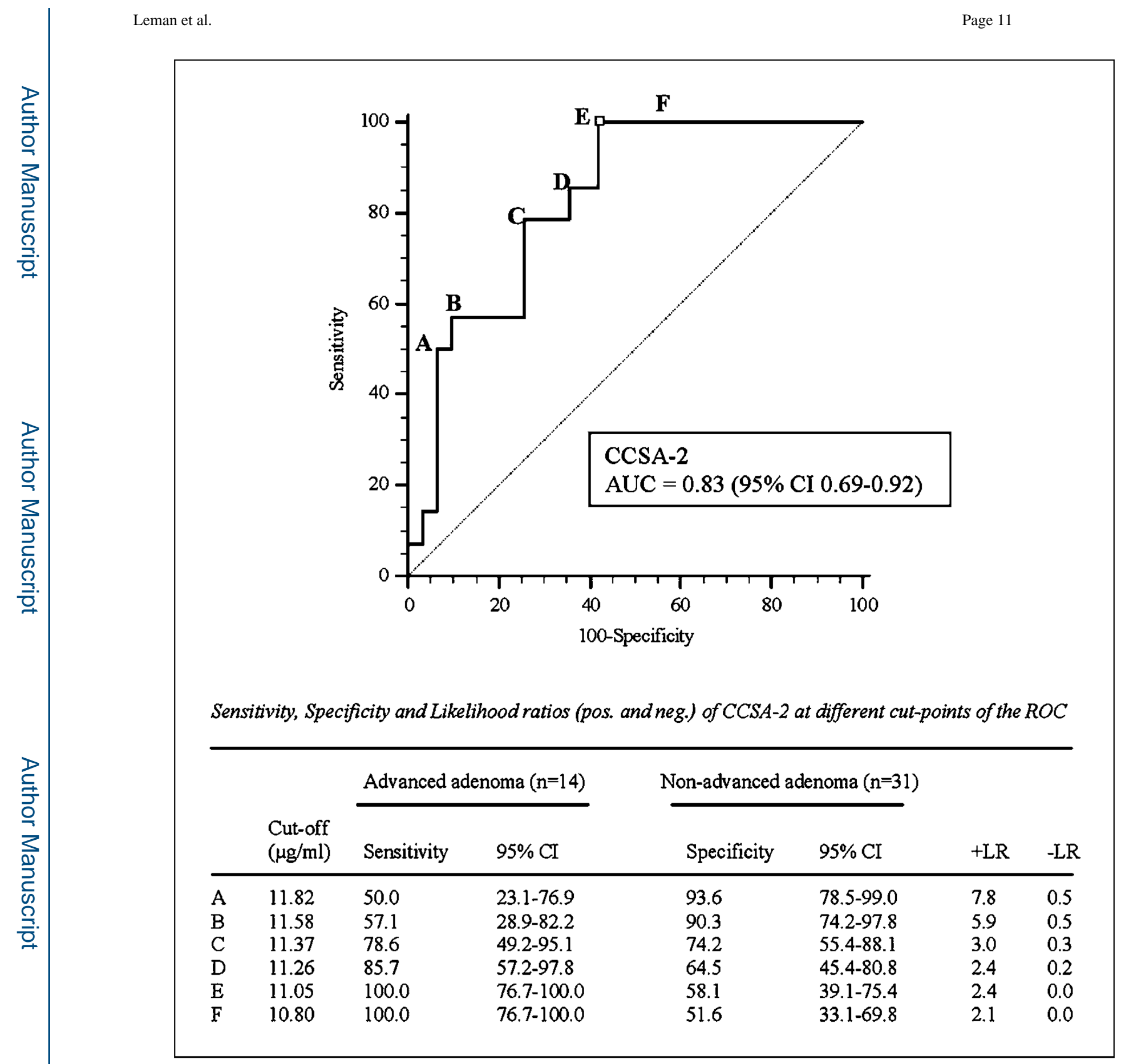

Fig. 4.

ROC curves forCCSA-2inseparating nonadvanced adenoma individuals from those with advanced adenomas. 


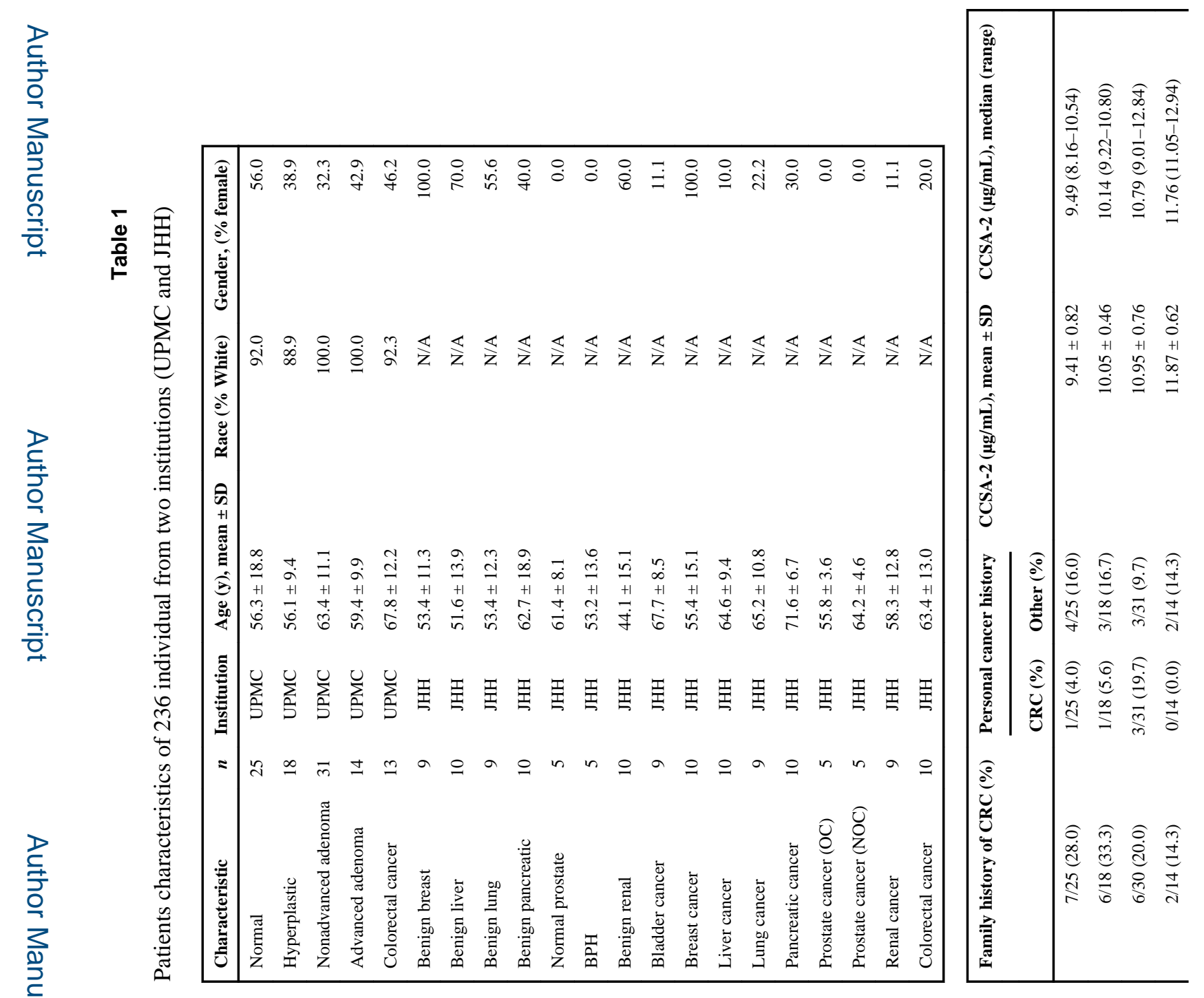

Clin Cancer Res. Author manuscript; available in PMC 2015 November 30. 


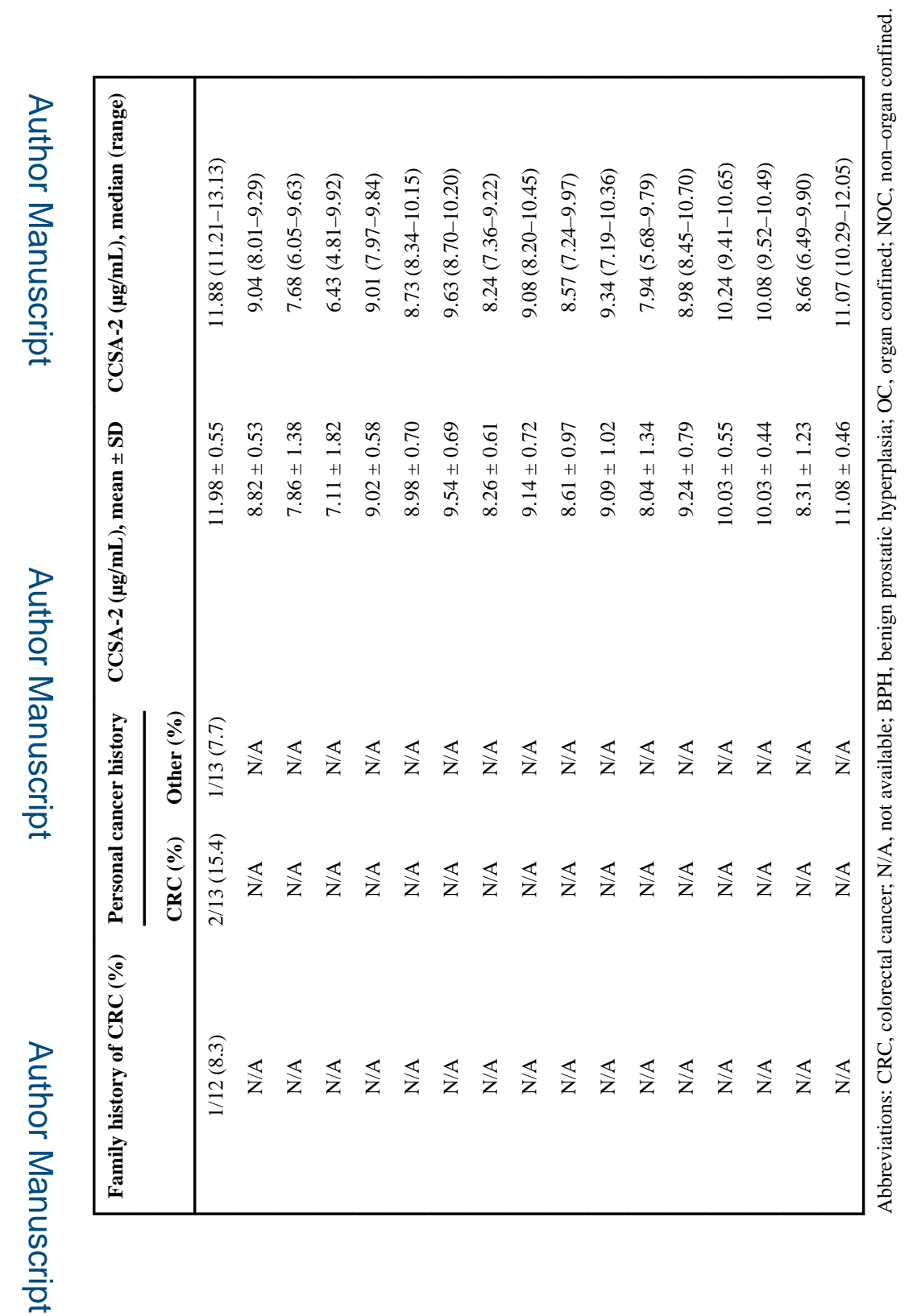

Clin Cancer Res. Author manuscript; available in PMC 2015 November 30. 
Table 2

Sensitivity and specificity for CCSA-2 at a specified cutoff ( $n=236)$

\begin{tabular}{|c|c|c|}
\hline \multirow[t]{2}{*}{ Cohort ${ }^{*}$} & \multicolumn{2}{|c|}{$\begin{array}{l}\text { Sensitivity CCSA-2 } \\
(10.8 \mu \mathrm{g} / \mathrm{mL} \text { cutoff })\end{array}$} \\
\hline & $n(\%)$ & $95 \% \mathrm{CI}$ \\
\hline Colorectal cancer & $22 / 23(95.6)$ & $78.1-99.8$ \\
\hline Advanced adenoma and colorectal cancer & $36 / 37(97.3)$ & $85.8-99.5$ \\
\hline Advanced adenoma & $14 / 14(100.0)$ & $76.8-100$ \\
\hline Nonadvanced adenoma and advanced adenoma & 29/45 (64.4) & $48.8-78.1$ \\
\hline \multirow[t]{2}{*}{ Cohort $^{\dagger}$} & \multicolumn{2}{|c|}{$\begin{array}{l}\text { Specificity CCSA-2 } \\
\text { (10.8 } \mu \mathrm{g} / \mathrm{mL} \text { cutoff })\end{array}$} \\
\hline & $n(\%)$ & $95 \% \mathrm{CI}$ \\
\hline Normal and hyperplastic & $42 / 43(97.7)$ & $87.7-99.9$ \\
\hline Normal, hyperplastic, and nonadvanced adenoma & $58 / 74(78.4)$ & $67.3-87.1$ \\
\hline Benign diseases & $58 / 58(100)$ & $93.8-100$ \\
\hline Other cancers & $67 / 67(100)$ & $94.6-100$ \\
\hline
\end{tabular}

